\title{
Ethics of Clinical Research Involving Adults with Impaired Decision-Making Capacity
}

\author{
Tonmoy Biswas \\ $5^{\text {th }}$ Year, MBBS, Faridpur Medical College, Bangladesh \\ Email: tshekhor@yahoo.com
}

\begin{abstract}
Background: Proper decision making capacity, adequate disclosure and voluntary decisions are basic constituents of informed consent which is required in surgical procedures, any interventions, any tissue collection, or any research involving the human participants. But, it becomes more hectic if the participants or patients are physically or mentally impaired for proper understanding or rational decision making. Time has gone by assembling or regulating effective laws for research involving persons with impaired decision making capacity. Still, question arises, is it ethical to enroll an incompetent person who is not physically or mentally fit to make a decision in risky research or interventional trials? If it is, how the informed consent and ethical measures can be taken? Method: Extensive literature review was done in Google scholar, PubMed and national or institutional websites with the corresponding keywords to summarize the cases of impaired decision making and regulation of informed consent and ethical measures in those cases. Results: Decision making capacity requires three level of capacities and four levels of abilities. If a person has factual understanding, implies a certain level of rational belief, knows to manipulate information to arrive at a choice and remains stable on the choice, is known to be capacitated in decision making. Impaired decision making capacity is more common in Alzheimer's disease and schizophrenia research. Although a definite line between decisional capacity and incapacity is still in question, many assessment tools are available to conclude it. Moreover, decisional incapacity has been found as a significant ratio in general or psychiatric hospitals and nursing homes regarding psychological disorders or critically ill conditions. But, these conditions should not prevent anyone from understanding, choosing, or accepting any intervention as sometimes they may have some preserved abilities too. As per accepted ethics, respect for persons incorporates at least two ethical convictions. First, the individual should be treated as an autonomous agent and second, the person with diminished autonomy is entitled to protection. That's why, in case of severe psychiatric diseases and Alzheimer's diseases, surrogate consent is recommended. But surrogacy should be reviewed by the institutional review board (IRB). Multimedia consent process, advanced consent directives, rational consent waiver and many other processes are practiced in case of ethical research involving decisional incapacitate persons which are discussed in the paper. Conclusion: It should be clarified by the IRB whether involvement of impaired subjects has beneficial scientific aim or not. Capacity assessment system should be in an organized and systemic way. Threshold for capacity and recognition of persons able to conduct this process should be fixed. Role of surrogacy and involvement of IRB to align it in a proper manner is always a matter of concern. Consideration of risk management, subjects' autonomy and assent-dissent issues should be clarified in research.
\end{abstract}

Key words: Research Ethics; Incapacity; Research Ethics Committee; Informed consent; Bangladesh 
Introduction: Ethical issues are often emphasized in research involving human participants as mandatory. Informed consent, as the most essential aspect of medical research, owes its origin to the father of medicine, Hippocrates. Currently, ethical principles and the consent have been shown to have great implications in clinical research, randomized control trial, medical intervention, treatment, and surgery. Informed consent is based on three model; information sharing, decisional capacity and volunteerism and requires four types of abilities for proper decision making. But, if the participants are adults (excluding pediatric and emergency patients) and suffering from some mental disorders, schizophrenia, Alzheimer disease, and critical illness or other handicapped condition, their decisional capacity is mostly impaired ${ }^{1,2}$. What is the way of consent in those cases? What are the problems specifically arise concerning this issue? What is the ethical solution to those problems? This paper will review answers to the mentioned questions and specific measures.

Method: This research was done during the three month course of National Institute of Health (NIH), USA on Ethical and Regulatory Aspects of Clinical Research conducted by Bangladesh Bioethics Society in 2014. Through the literature review from Google scholar, PubMed and national or institutional websites, it has been wanted to focus on history and consequence of research involving decisionally incompetent persons with highlighting ethics implemented in different research and practical studies. Research principles involving patients of Alzheimer's disease, schizophrenia, patients of intensive care unit, of various psychological disorders, and with other decisional incapacities have been emphasized and reviewed by corresponding key-words. Pediatric research and emergency research is to be excluded in this research article.

Decisional Capacity and Incapacity: Decisional capacity defines a person's abilities to realize, appreciate, reason, and make a selection ${ }^{3}$. It comprises evaluating a patient in a clinical care or subject in a research whether he/she is psychologically or legally skillful of adequate decisionmaking or not ${ }^{4}$. Specifically, four levels of abilities counting as understanding, appreciating, reasoning, and communicating a stable choice are required to evaluate a person as decisionally capacitate. Clinically, decisional capacity is closely related to competence that means the ability to consent for a care, management, and treatment or ability to refuse those ${ }^{5}$. However, it is task based process, that is, a person has or lacks capacity for a particular decision at a particular time and under particular situations. On the other hand, defining decisional incapacity is not measured by definite objective standards, but on the cautious judgment of those who are closely related to that person and have a proper understanding about him $/$ her $^{6}$. Therefore, a patient or subject has the deficient capacity related to a matter, if at specific time he cannot make a decision for himself related to that matter as a result of an impairment or functional trouble of the mind or brain ${ }^{7}$.

Thus, healthcare providers or clinical researchers should be observant to hints of decisional incapacity during assumption. It should be kept in mind that disagreement with the clinician's treatment or recommendation is not itself only a clue to reduced capacity anyway. In various 
conditions, a person can be incompetent in decision making, such as Alzheimer disease, schizophrenia, some mental and psychiatric disorders, neurological disorders, or some other medical conditions ${ }^{4,6}$. Additionally, a person can be decisional incapacitate due to over-staying in intensive care unit, post-operative state, medical effects, persistent distracting pain, and loos, grief, or devastating news etc 6 .

The principle of autonomy involves a physician's consideration of the authority of a patient to decide, even if the decisions seem to be unwise. Therefore, an individual must have decisionmaking capacity for making an autonomous decision or giving an informed consent to any medical treatment or research participation ${ }^{4}$.

Problem historical scenario: Though the autonomy of a patient was first talked about in 1767, the idea of informed consent was in an ambiguous manner for many years ${ }^{8}$. In 1898, Albert Neisser, discoverer of the gonococcus was first questioned about ethical issues and informed consent when he injected cell free serum from syphilitic patients to non-syphilitic patients. Though, according to Albert Moll, about 600 cases of unethical research without informed consent were reported, the case of Neisser could trigger a firing debate in public society in that time. In 1900, Prussian parliament issued an ethical regulation for bio-medical research which was the first interference in the open research involving human participants ${ }^{9}$. Regarding a case in 1914, a judge in New York ruled to take patients' consent before surgery although he withdrawn the necessity of consent in emergency cases and in cases of unconscious patients as an exception. Consequently, after some more debates and events, German law addressed the ethical matter of therapeutic and non-therapeutic research in 1931. This was the first authorized regulation before the Nuremberg code in 1947 and declaration of Helsinki in 1964. However, from 1950 to 1970s different courts reshaped the ideas of informed consent and constructed it into a modern manner later on ${ }^{8,9}$. Informed consent was taken into concern in different times in the history in different places due to debated cases in 1971, 1972, 1973, 1974, 1976, 1978, 1980, 1984, 1986 and later years ${ }^{10}$.

Alzheimer Disease: Alzheimer disease (AD) is a neurological disorder which progressively abolishes memory and other mental functions caused by brain atrophy and spontaneous nerve tissue degeneration. It usually starts with forgetfulness and confusion and progress into losing all types of memory with disorientation, ${ }^{11}$ mood swing, changes in sleep, mis-identification, agitation, depression, delusion, 12, 13 impaired speaking and writing, diminished thinking, reasoning, and decisional capacity ${ }^{11}$. As this condition lacks decisional capacity, it has been taken into a great concern to obtain informed consent. One study in United States showed 84\% impairment of at least one of the four abilities of decision making among the Alzheimer patients ${ }^{14}$. Another study in California showed worse performance of these patients in decision making even than schizophrenia and Diabetes patients ${ }^{15}$. About $60 \%$ of the Alzheimer disease (AD) patient was incompetent in decision making as reported in another research ${ }^{16}$. Likewise, in another study, $40 \%$ of the patients with $\mathrm{AD}$ were incapable to give consent in clinical trials and that is obviously 
significant in any basis ${ }^{17}$. All the data from research about ability of decision making among AD patients clearly states that in this condition, most of the patients don't have the sufficient acceptable understanding, appreciating, reasoning, and communicating capacity which is required for agreement with any informed consent for any intervention, treatment or clinical trial.

Schizophrenia: Schizophrenia is a chronic mental condition characterized by hallucination, illusion, delusion and impaired decision making ${ }^{18}$ caused by unclear genetic malfunction and environmental factors ${ }^{19}$. Some other features of schizophrenia include disorganized motor functions and thinking ${ }^{18}$. Decision making in the schizophrenia is significantly worse than other healthy participants due to lack of working memory in all age groups, ${ }^{20}$ even in the early stages of adolescence $^{19}$. A study showed that $52 \%$ of their schizophrenia patient was impaired in decision making where it was only $12 \%$ in angina patients and $4 \%$ in non-psychiatric patients ${ }^{22}$. Also, in a study, $18-20 \%$ schizophrenia patients had extreme low understanding and reasoning level. ${ }^{23}$ Schizophrenia in younger patients can result fewer years of education than non-psychiatric young patients $^{24}$. Even, their decisional capacity was found lower than the HIV patients ${ }^{25}$. So, it is certain that during treatment or management of a schizophrenia patient or in case of any clinical trial, informed consent is a matter of unease due to decisional incapacity of most of the patients.

Other incapacities: Depending upon the affected area, stroke patients are sometimes incapable of proper decision making and informed consent ${ }^{26}$. Roughly, about 20 to $25 \%$ of the psychiatric patients are in lack of at least one component of decisional capacity ${ }^{27}$. One research among the medical patients in hospital showed $48 \%$ incapacity for informed consent among variety of medical conditions which signifies the ethical matter to involve them in any research or clinical $\operatorname{trial}^{28}$. Another study in a nursing center showed about $30 \%$ incompetency among different medical situations ${ }^{29}$. In a study among the Parkinson disease patients with impaired cognition, most were not capable to give informed consent: only $13 \%$ were capable in that drug trial ${ }^{30}$. Patient of Intensive care unit most of the times remain unconscious and cannot give any consent mostly ${ }^{31}$. Although chief conditions have been discussed, there can be many other causes of decisional incapacity for informed consent in adult as discussed before. Finally, informed consent and other ethical matters become greatly unstable in medical treatment, surgery, clinical trial, and research studies involving human participants if they are incapable of understanding, reasoning, appreciating and communicating, concisely of decision making.

Capacity evaluation procedure: Basically, there are three vital steps for capacity assessment of adult participant: First, to detect the information related to the decision by examining the decision that needs to be anticipated, as well as the characteristics of substitute rational decisions with the pros and cons of them; Second, to assess the cognitive ability for evaluating capability of understanding the information, decision making, and volunteerism; Third, to assess the factors acting behind one's capacity like delusions, hallucinations, depression, manic illnesses, and lack of maturity ${ }^{32}$. MacArthur Competence Assessment Tools for Clinical Research and Treatment 
(MacCAT-CR and MacCAT-T) is widely used for capacity assessment. Moreover, Competency to Consent to Treatment Inventory (CCTI), and Mini-Mental State Examination (MMSE) are also necessary tools in research involving adult participants ${ }^{33}$. Other tools include Aid to Capacity Evaluation (ACE), the Hopkins Competency Assessment Test (HCAT), and the Understanding Treatment Disclosure (UTD) etc ${ }^{34}$. Although, there are some debates and counter-opinions based on of evaluation of the assessment measures and tools ${ }^{35}$, this paper will not discuss that issue.

Preserved abilities: In a previous research, it was reported that $38 \%$ of those judged incapable of consenting to drug Randomized Control Trial (RCT) and 55\% of those judged incompetent of consenting to neurosurgical RCT are still capable of appointing a prox $\mathrm{y}^{36}$. If this the scenario, then participants should be first asked to appoint an individual as surrogate themselves before further deterioration of their cognitive thinking and rational capacity.

Ethical approaches to the problems: Ethical approaches regarding informed consent in decisional incapacitated adults are still in mirage; no exact policy addressed this problem.

Alzheimer disease ethics: In any study involving Alzheimer disease patient, it is better to check for decisional capacity properly, as some cases may have intact decisional capacity as exception. If the capacity is found diminished, it is recommended to check for the preserved abilities to appoint a family member or known person as a surrogate himself. But, in the matter of research interventions, it is better to make a proxy surrogate at the early stage of disease progression before full loss of capacity ${ }^{37}$. This type of research protocol is highly screened by institutional review boards (IRBs) and they accept surrogate consent most of the cases. But, the acceptance of person working as proxy varies. A study in USA showed that most of IRB were affirmative in appointment of authorized representatives, spouses, parents, adult children, and adult siblings as a surrogate but negative in case of adult grandchildren, other adult families, and friends. But, in case of this kind of research, assessment of direct benefits from the expected results and probable risks are measured and evaluated effectively. Most review boards permit the research if it has minimal tolerable risks, otherwise don't ${ }^{38}$.

Ethics in Schizophrenia: The approaches in a case of schizophrenia also include the initial step of Alzheimer disease that is the proper assessment of decisional capacity and calculation of preserved abilities. However, in schizophrenia, Informed consent can be theorized as comprising three basic elements: information sharing, decisional capacity and capacity for voluntarism ${ }^{39}$. The first one is information sharing; all the information regarding the trial or study should be disclosed to the patient including study aims, possible risks and advantages of participation, options of participation, and related study plan like using a placebo ${ }^{40}$. To address the cognitive impairment, multimedia presentation and computerized information with spontaneous feedback showed significant increase in decisional capacity in these patients ${ }^{41}$. But, problem arises with the second element which is decisional capacity and as we discussed, schizophrenia patients often lack of it. 
To solve this matter, we can use surrogate consent like Alzheimer but, most schizophrenia patient prefer autonomous decision making rather than proxy consent ${ }^{42}$. So, Surrogacy should always be the last option. To deal with their cognitive impairment, advanced research directives can be the preference. It is actually a written prior document that provides clear consent about the all upcoming possibilities. It will indicate the entire possible scenario that may arise during the course of research, involving risks and benefits of each step. This is advanced strategy to preserve the autonomy of the schizophrenic patients in decision making despite having some limitations ${ }^{43}$. In case of its limitations, surrogate can be chosen as an option. Finally comes the third element of informed consent, voluntarism which is more important but less understood. Voluntarism is always in question when the physician treating the individual is himself an investigator because the individual depends upon the clinician for continuous well-being. But, this problem can be solved by involving the researchers who are not related to the patient's treatment ${ }^{44}$. Another useful strategy is to encourage schizophrenia community in research procedure and to involve them in reviewing the study plan. Web based tool to enhance the informed consent process in schizophrenia is also a newly appreciated theory ${ }^{45,46}$.

Ethics in other Psychiatric Conditions: In psychiatric conditions, research undergoes more ethical evaluation. As there are two types of research, therapeutic and non-therapeutic; nontherapeutic has fewer risks. So, it can be permitted after proper assessment, but the therapeutic study requires more concern ${ }^{47}$. In case of proper direct beneficial study, IRB can determine the legally authorized surrogate. If the risk is more than the minimal, the consent of the team involved in patient treatment must be obtained ${ }^{48}$. It is recommended to have some core safeguards in psychiatric research; IRB risk-benefit assessment, consent assessment, necessity requirement, sufficient evidences with proxy decision makers, assent-dissent issues, and independent monitoring. More ethical emphasize should be given on blood draw, two PET (Positron Emission Tomography) scans with arterial line, drug withdrawal, and phase-II drug trial ${ }^{49}$. However, consent waivers sometimes can also be considered in psychiatric research based on some criteria in certain cases but those should be appropriately judged by the IRB ${ }^{50}$.

Ethics of consent in critically ill or intensive care unit (ICU) patients: In the critically ill patients, informed consent also requires its basic three components as mentioned above. But, it is predictive that most participants are not capable to fulfill the criteria of informed consent at this stage. However, information sharing, decisional capacity and capacity for voluntarism can be addressed consciously before progression of alignment. If it is not possible, surrogate consent is often practiced ${ }^{51}$. But study showed that surrogates were more inaccurate regarding similarities with the patient choice ${ }^{52}$. So, researcher also looked for other measures. Sometimes, the specific informed consent was not obtained ${ }^{53}$. Some regulations also suggested waiver of consent in emergency cases as an exception. In some cases like septic shock, waiver of informed consent did not have negative effect upon the progression of research. In recent years, some ethical regulations have been agreed to become positive in waiving consent procedure of clinical trial for critical 
patients in intensive care units. But for that case, proper assessment of risks by IRBs is mandatory ${ }^{54}$.

Conclusion: Informed consent of the incapacitate adults in decision-making is an emerging ethical issue in health research. At first we have to determine a definite line between decisional capacity and incapacity and estimate the capabilities of the participant with the established assessment tool with proper observation of the preserved abilities. According to the statistical data, Alzheimer patients, schizophrenia patients, psychiatric patients, and critically ill patients are invariably unable to understand ethical matters of treatment or research and unable to give informed consent. We should acquire data from them by the methods described above. Established guidelines are needed to be followed to maintain participants' autonomy and beneficence. After that purpose of the research can be served ethically. Revision of national medical ethics policies by the experts regarding this issue is mandatory. Further, health professionals should be informed about the serious ethical matter. However, appropriate ethical approaches to the decisional incapacitate adults is solely obligatory to heighten the practicability of research involving human participants.

\section{References:}

1. Koch Hans-Georg. Reiter-TheilStella, Helmchen Hanfried. Informed Consent in Psychiatry: European Perspectives of Ethics, Law and Clinical Practice, IST ed. BadenBaden: Nomos Verl-Ges.Germany, 1996.

2. Wood Susan Y, Friedland Barbara A, McGrory C. Elizabeth. Informed Consent: From Good Intentions to Sound Practices A Report of a Seminar. Population Council New York 2001; www. popcouncil.org [Ref list]

3. Appelbaum PS, Grisso T. MacArthur Competence Assessment Tool for Clinical Research (MacCAT-CR) Sarasota: Professional Resource Press; 2001.

4. Ethics Fast Fact; UCSF School of Medicine; http://missinglink.ucsf.edu/lm/ethics/Content\%20Pages/fast_fact_competence.htm; Last accessed 12 December, 2014

5. Jonsen et al. Clinical Ethics: A Practical Approach to Ethical Decisions in Clinical Medicine, 7 th

edition. Viewed 12 December 2014.

http://accessmedicine.mhmedical.com/book.aspx?bookId=364

6. Ethics Committee Consortium of the Center for Practical Bioethics; Guidelines for the Determination of Decisional Incapacity; http://ethics.missouri.edu/docs/Guidelines $\% 20$ for $\% 20$ Determination $\% 20$ of $\% 20$ Decisiona 1\%20Incapacity.pdf ; Last accessed 12 December, 2014

7. Mental capacity act 2005; UK Legislation; <http://www.legislation.gov.uk/ukpga/2005/9/section/2>; Last accessed December 12, 2014

8. Rose, Roger M. M.D.; INFORMED CONSENT: HISTORY, THEORY, AND 
PRACTICE.; American Journal of Otology: January 1986 - Volume 7 - Issue 1 - ppg 8285

9. Jochen Vollmann, Rolf Winau; Informed consent in human experimentaton before the Nuremberg code; BMJ 1996;313:1445-7

10. Murray, Peter M. "The history of informed consent." The Iowa orthopaedic journal 10 (1990): 104.

11. Alzheimer Disease; Mayo-Clinic; $\quad \underline{\text { http://www.mayoclinic.org/diseases- }}$ conditions/alzheimers-disease/basics/definition/con-20023871; Last accessed: December 14, 2014

12. Alzheimer Disease; Web MD; http://www.webmd.com/alzheimers/guide/alzheimersdisease-symptoms; Last accessed: December 14, 2014

13. Devanand, D. P., et al. "The course of psychopathologic features in mild to moderate Alzheimer disease." Archives of general psychiatry 54.3 (1997): 257-263.(Abstract)

14. Kim, Scott YH, et al. "Assessing the competence of persons with Alzheimer's disease in providing informed consent for participation in research." American Journal of Psychiatry 158.5 (2001): 712-717.

15. Palmer, Barton W., et al. "Assessment of capacity to consent to research among older persons with schizophrenia, Alzheimer disease, or diabetes mellitus: comparison of a 3item questionnaire with a comprehensive standardized capacity instrument." Archives of General Psychiatry 62.7 (2005): 726-733.

16. Karlawish, J. H. T., et al. "The ability of persons with Alzheimer disease (AD) to make a decision about taking an AD treatment." Neurology 64.9 (2005): 1514-1519.

17. Jefferson AL, et al. Decisional capacity for research participation in individuals with mild cognitive impairment. J Am Geriatr Soc. 2008;56:1236-1243.

18. Schizophrenia; Mayo- Clinic; $\quad$ http://www.mayoclinic.org/diseasesconditions/schizophrenia/basics/ definition/con-20021077; Last accessed: December 14, 2014

19. Schizophrenia; Web MD; http://www.webmd.com/schizophrenia/guide/default.html ; Last accessed: December 14, 2014

20. Heerey, Erin A., Kimberly R. Bell-Warren, and James M. Gold. "Decision-making impairments in the context of intact reward sensitivity in schizophrenia." Biological psychiatry 64.1 (2008): 62-69.

21. Kester, Hana M., Serge Sevy, Eldad Yechiam, Katherine E. Burdick, Kelly L. Cervellione, and Sanjiv Kumra. "Decision-making impairments in adolescents with early-onset schizophrenia." Schizophrenia research 85, no. 1 (2006): 113-123.

22. Jeste DV, Depp CA, Palmer BW. Magnitude of Impairment in Decisional Capacity in People With Schizophrenia Compared to Normal Subjects: An Overview. Schizophrenia Bulletin 2006;32(1):121-128. doi:10.1093/schbul/sbj001.

23. Grisso T, Appelbaum PS. Comparison of standards for assessing patients' capacities to make treatment decisions. Am J Psychiatry. 1995;152:1033-1037. 
24. Dunn LB, Lindamer LA, Palmer BW, Golshan S, Schneiderman LJ, Jeste DV. Improving understanding of research consent in middle-aged and elderly patients with psychotic disorders. Am J Geriatr Psychiatry. 2002;10:142-150.

25. Moser D, Schultz S, Arndt S, Benjamin ML, Fleming FW, Brems CS, Paulsen JS, Appelbaum PS, Andreasen NC. Capacity to provide informed consent for participation in schizophrenia and HIV research. Am J Psychiatry. 2002;159:1201-1207.

26. White-Bateman SR, Schumacher HC, Sacco RL, Appelbaum PS. Consent for thrombolysis in acute stroke: review and future directions. Arch Neurol 2007;64:785-792

27. Vollmann J, Bauer A, Danker-Hoipfe H, Helmchen H. Competence of mentally ill patients: a comparative empirical study. Psychol Med 2003;33:1463-1471

28. Raymont V, Bingley W, Buchanan A, et al. Prevalence of mental incapacity in medical inpatients and associated risk factors: cross-sectional study. Lancet 2004;364:1421-1427

29. Barton Jr, C. Dennis, Harminder S. Mallik, William B. Orr, and Jeffrey S. Janofsky. "Clinicians' judgement of capacity of nursing home patients to give informed consent." Psychiatric services (Washington, DC) 47, no. 9 (1996): 956-960.

30. Karlawish, J., Cary, M., Moelter, S. T., Siderowf, A., Sullo, E., Xie, S., \& Weintraub, D. (2013). Cognitive impairment and PD patients' capacity to consent to research. Neurology, 81(9), 801-807. doi:10.1212/WNL.0b013e3182a05ba5

31. Booth, M., Doherty, P., Fairgrieve, R., \& Kinsella, J. (2004). Relatives' knowledge of decision making in intensive care. Journal of Medical Ethics, 30(5), 459-461. doi:10.1136/jme.2002.000802

32. Amer, A. B. (2013). Informed Consent in Adult Psychiatry. Oman Medical Journal, 28(4), 228-231. doi:10.5001/omj.2013.67

33. Dunn, L. B., \& Misra, S. (2009). Research Ethics Issues in Geriatric Psychiatry. The Psychiatric Clinics of North America, 32(2), 395-411. doi:10.1016/j.psc.2009.03.007

34. Laura L. Sessums, JD, MD ; Assessing Medical Decision-Making Capacity; Physician's Weekly; Feature article May 8, 2012; http://www.physiciansweekly.com/medical-decisionmaking-capacity/; Last accessed: December 15, 2014

35. Kim, Scott YH. Evaluation of capacity to consent to treatment and research. Oxford University Press, 2009.

36. Kim, Scott YH, Jason H. Karlawish, H. Myra Kim, Ian F. Wall, Andrea C. Bozoki, and Paul S. Appelbaum. "Preservation of the capacity to appoint a proxy decision maker: implications for dementia research." Archives of general psychiatry 68, no. 2 (2011): 214219.

37. Kim, S. Y. H. (2011). The ethics of informed consent in Alzheimer disease research. Nature Reviews. Neurology, 7(7), 410-414. doi:10.1038/nrneurol.2011.76

38. Gong, M. N., Winkel, G., Rhodes, R., Richardson, L. D., \& Silverstein, J. H. (2010). Surrogate consent for research involving adults with impaired decision making: Survey of Institutional Review Board practices. Critical Care Medicine, 38(11), 2146-2154. doi:10.1097/CCM.0b013e3181f26fe6 
39. Roberts L W. Ethics and mental illness research. Psychiatr Clin North Am 2002. 25525545.545

40. Macklin R. Understanding informed consent. Acta Oncol 1999. 3883-87.87

41. Dunn L B, Lindamer L A, Palmer B W. et al Improving understanding of research consent in middle-aged and elderly patients with psychotic disorders. Am J Geriatr Psychiatry 2002. 10142-150.150

42. Roberts L W, Warner T D, Brody J L. Perspectives of patients with schizophrenia and psychiatrists regarding ethically important aspects of research participation. Am J Psychiatry 2000. 15767-74.74

43. Arboleda-Florez J, Weisstub D N. Ethical research with the mentally disordered. Can J Psychiatry 1997. 42485-491.491

44. Levine R J. Consent issues in human research. In: Emanuel EJ, Crouch RA, Arras JD, et al eds. Ethical and regulatory aspects of clinical research: readings and commentary. Baltimore, MD: Johns Hopkins University Press, 2003. 197-201.201

45. Macaulay A C, Commanda L E, Freeman W L. et al Participatory research maximises community and lay involvement. BMJ 1999. 319774-778.778

46. Harmell, A. L., Palmer, B. W., \& Jeste, D. V. (2012). Preliminary Study of a Web-based Tool for Enhancing the Informed Consent Process in Schizophrenia Research. Schizophrenia Research, 141(2-3), 247-250. doi:10.1016/j.schres.2012.08.001

47. Welie SP \& Berghmans RL: Inclusion of patients with severe mental illness in clinical trials: issues and recommendations surrounding informed consent. CNS Drugs 2006; 20:67-83.

48. S. J. Delano, J. L. Zucker et al. Protecting mental health research subjects without prohibiting progress. Hosp Community Psychiatry. 1994 June; 45(6): 601-603.

49. Wendler D, Prasad K. Core Safeguards for Clinical Research with Adults Who Are Unable To Consent. Ann Intern Med. 2001;135:514-523. doi:10.7326/0003-4819-135-7200110020-00011

50. Rosoff AJ. Informed consent (a guide for health care providers). Rockville, Maryland: Aspen Publications; 1981;P14.

51. Davis N, Pohlman A, Gehlbach B, et al. Improving the Process of Informed Consent in the Critically Ill. JAMA. 2003;289(15):1963-1968. doi:10.1001/jama.289.15.1963.

52. Coppolino, Michael, and Lynn Ackerson. "Do surrogate decision makers provide accurate consent for intensive care research?." CHEST Journal 119, no. 2 (2001): 603-612.

53. Chenaud, C., Merlani, P., Luyasu, S., \& Ricou, B. (2006). Informed consent for research obtained during the intensive care unit stay. Critical Care, 10(6), R170. doi:10.1186/cc5120

54. Chenaud, C., Merlani, P., \& Ricou, B. (2007). Research in critically ill patients: standards of informed consent. Critical Care, 11(1), 110. doi:10.1186/cc5678

\section{Author declared no conflict of interest.}

